\title{
Low Profile Frequency Reconfigurable PIFA Antenna using Defected Ground Structure
}

\author{
Pham Trung Minh ${ }^{1,2}$, Nguyen Trong Duc ${ }^{2}$, Phan Xuan Vu ${ }^{1}$, Nguyen Thanh Chuyen ${ }^{1}$, Vu Van Yem ${ }^{1}$ \\ ${ }^{1}$ School of Electronics and Telecommunications, Hanoi University of Science and Technology, Vietnam \\ 2 Faculty of Information Technology, Vietnam Maritime University, Vietnam
}

Correspondence: Pham Trung Minh, minhpt@vimaru.edu.vn

Communication: received 4 January 2017, revised 11 May 2017, accepted 8 July 2017

Online publication: 30 October 2017, Digital Object Identifier: 10.21553/rev-jec.153

The associate editor coordinating the review of this article and recommending it for publication was Dr. Vo Nguyen Quoc Bao.

\begin{abstract}
In this paper, we design and implement a low profile frequency reconfigurable Planar Inverted-F Antenna (PIFA) for WLAN, m-WiMAX and UMTS applications. Different from several conventional designs, the air layer in our antenna is removed, while the radiator patches and the ground plane are printed on two sides of the same substrate. This makes the antenna structure thin and lightweight. The defected ground structure (DGS) and coplanar sorting-trips are also designed for adjusting lower operating frequencies without increasing the antenna's size. Three PIN-diodes are used in appropriate positions for accurate switches between frequency bands. Moreover, the three radiator patches' parameters are optimally selected on all configurations using Genetic Algorithm (GA). Simulation results show that depending on the ON/OFF states of the PIN-diodes, the antenna can operate in three applicable frequency bands, i.e., $2.1 \mathrm{GHz}, 2.4 \mathrm{GHz}$, and $3.5 \mathrm{GHz}$ with the corresponding peak gains of $0.48 \mathrm{dBi}, 3.55 \mathrm{dBi}$, and $4.33 \mathrm{dBi}$. The antenna occupies an overall size of $63.5 \times 33.5 \times 1.6$ $\mathrm{mm}^{3}$, which can be easily fabricated and integrated into small wireless devices. Simulated and measured results are also compared to validate the correctness of the antenna design.
\end{abstract}

Keywords- DGS, frequency reconfigurable antenna, optimization algorithm, PIFA, PIN-diode.

\section{INTRODUCTION}

The rapid development of wireless technologies requires communication equipments to be small, compatible and multi-functional. They need to be applicable with different communication standards, such as wireless local area network (WLAN), mobile worldwide interoperability for microwave access (m-WiMAX) etc... In order to meet these requirements, the devices' antennas are expected to be multi-band or frequency reconfigurable $[1,2]$. In addition, the devices need to be compact and capable of a flexible frequency conversion. Among existing solutions, frequency reconfigurable antenna design has attracted much attention recently [3].

Nowadays, the planar inverted F-antenna (PIFA) is widely implemented in wireless communication thanks to its simplicity, low cost, and low profile [4-6]. The antenna is also investigated in the literature of frequency reconfigurable design. In [7], a PIN-diode and a tunable varactor are implement simple antenna with the size of $70 \times 30 \times 9 \mathrm{~mm}^{3}$. By switching the PINdiode and turning the varactor, the antenna can operate on frequency bands of United States personal communications services (USPCS), WLAN, wideband code division multiple access (WCDMA), and m-WiMAX with gains of $2.84,1.49,2.81$, and $1.25 \mathrm{dBi}$, respectively. In [8], the proposed multi-layer antenna occupies a size of $179 \times 155 \times 9.5 \mathrm{~mm}^{3}$. The antenna uses 13 Radio Frequency MicroElectroMechanical Systems (RF
MEMS) switches to achieve five bands of 0.221, 0.47, $0.62,0.935$, and $4.96 \mathrm{GHz}$. Another reconfigurable PIFA antenna with 8 shapes is reported in [9], which occupies an overall size of $40 \times 40 \times 3 \mathrm{~mm}^{3}$. The antenna can be switched between two frequency bands of $2 \mathrm{GHz}-$ $3 \mathrm{GHz}$ and $4 \mathrm{GHz}-7 \mathrm{GHz}$. Nevertheless, the structures of all above designs are on stacks so that there always exist an air layer between the radiator and the ground plane connected by shorting walls or shorting pins. As a result, these antennas might result in a high profile with a thickness of over $3 \mathrm{~mm}$, which might not suitable for nowadays modern mobile devices.

To cope with the high profile, several works have been carried out. In particular, in order to remove the air layer, the ground plate and radiation patches are set to be coplanar as described in [10] and [11] with overall sizes of $80 \times 40 \times 1.6 \mathrm{~mm}^{3}$ and $110 \times 60 \times 1.6$ $\mathrm{mm}^{3}$, respectively. In another case, a design in [12] with a uniplanar structure is printed on a substrate, and has a size of $115 \times 40 \times 1.6 \mathrm{~mm}^{3}$. These antennas are shown to have advantages in term of the antenna size in comparison with conventional works. Nevertheless, in the design process, antenna parameters are usually selected in a heuristic manner without any optimization approaches. Because of this reason, we believe that these antennas' overall size could not have been further optimized.

The optimization of the reconfigurable antenna parameters, in fact, have been mentioned in several articles 
[13-17]. In these works, the parameters are selected in each configuration separately thanks to different techniques such as Particle Swarm (PS) [13], Neural Network [14], Clonal Selection Algorithm (CSA) [15] and Genetic Algorithms (GA) [16, 17]. Nevertheless the optimization process should be taken into account on all configurations simultaneously. Therefore, it would be necessary and interesting to study the optimization process for all configurations simultaneously.

In this paper, based on the GA optimization technique, we design a new frequency reconfigurable PIFA antenna that is compact, easy to fabricate, and with enhanced bandwidth. The antenna can operate at wireless frequency bands of $2.1 \mathrm{GHz}, 2.4 \mathrm{GHz}$, and $3.5 \mathrm{GHz}$. Three PIN-diodes are used to satisfy predefined reconfiguration requirements. The proposed antenna has a low profile with the overall size of $64.5 \times 32.5 \times$ $1.6 \mathrm{~mm}^{3}$, while the radiator plane only occupies a size of $33.5 \times 18.5 \mathrm{~mm}^{2}$. It is important to note that this works is an extension of that in [18] with several design improvements to reduce the antenna size. First, the ground plane and the radiator patches are printed on both sides of a substrate in stead of only one side. Second, Defected Ground Structure (DGS) technique [19] is employed on the ground plane to achieve the design requirements more accurately. Simulated and measured results are also compared to validate the correctness of our work.

The rest of this paper is organized as follows. Section 2 presents fundamental issues related to the antenna design process. In Section 3, measurements and simulation results from our design are reported and discussed. Section 4 concludes our work.

\section{Design of Reconfigurable PIFA Atenna}

\subsection{Theory of PIFA}

Planar inverted-F antenna is a modification of the monopole antenna and the microstrip patch antenna, as shown in Figure 1. PIFA includes a radiator plane placed in parallel with a ground plane. A short circuit connects the radiator plane to the ground plane, and an antenna feeding mechanism for radiator patch. The fundamental mode resonant frequency for PIFA is given by [20]:

$$
f_{r}=\frac{c}{4 \sqrt{\varepsilon_{e f f}}(w p+h p-w s t-h)},
$$

where $c$ is the speed of light in free space, wst is the width of the shorting wall, $w p$ and $h p$ are dimensions of the radiator plane, $h$ is the thickness of the dielectric substrate and $\varepsilon_{e f f}$ is the effective permittivity of the medium between radiator plane and ground plane. The effective permittivity $\left(\varepsilon_{e f f}\right)$ is approximated by:

$$
\varepsilon_{e f f} \approx \frac{\varepsilon_{r}+1}{2}
$$

where $\varepsilon_{r}$ is the relative permittivity of the substrate material.

From (1) and (2), the operating frequency of the PIFA is affected by the dimensions of the radiation plate,

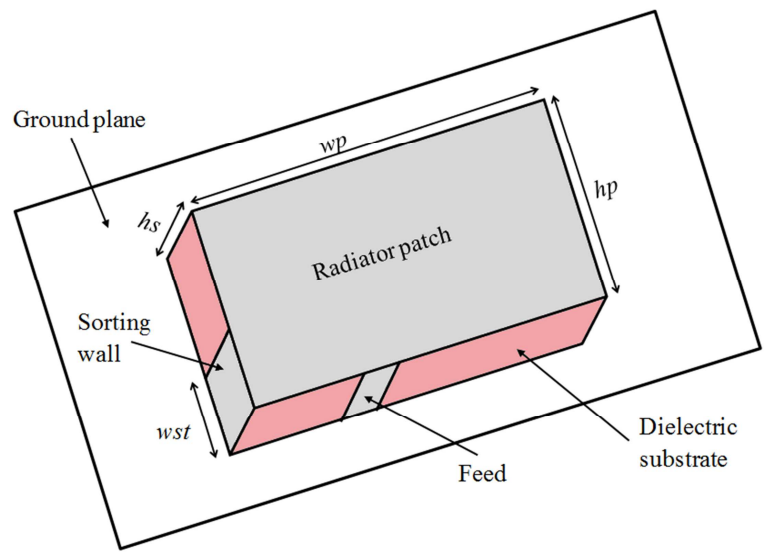

Figure 1. Geometry of a PIFA.
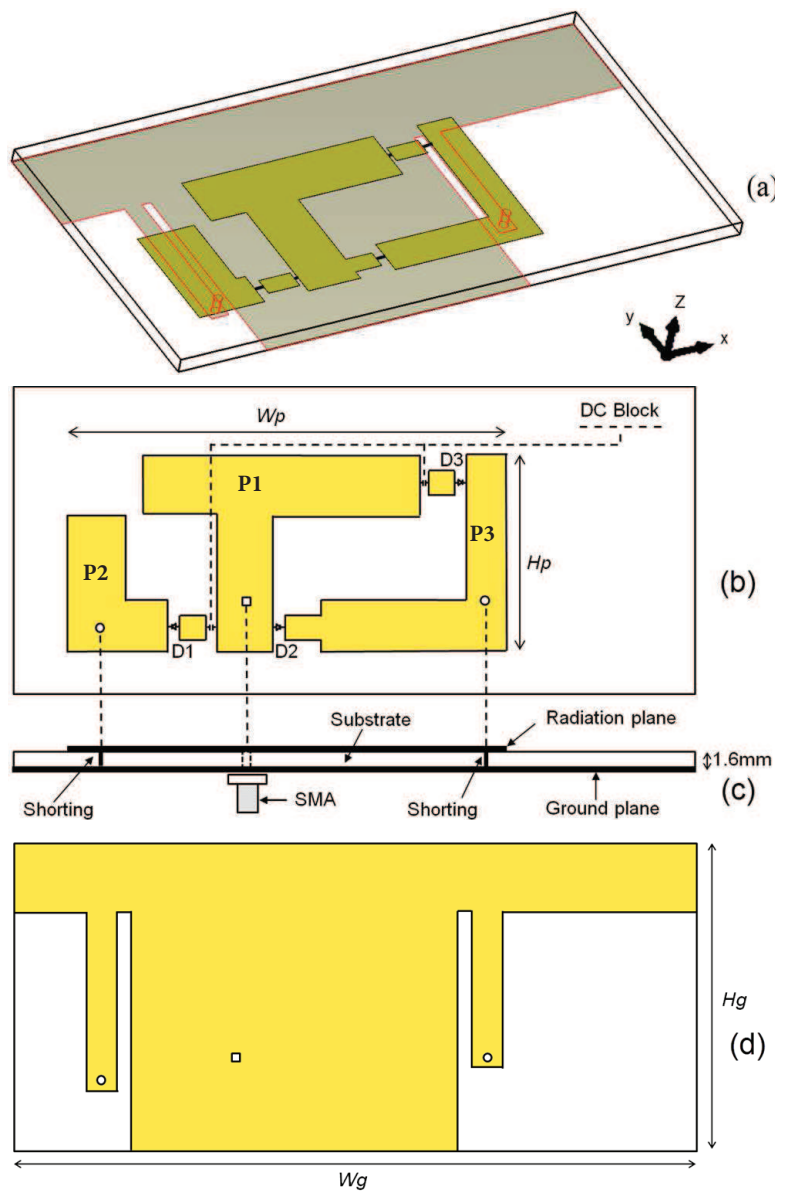

Figure 2. Structure of the proposed reconfigurable PIFA antenna (a) 3-D view, (b) Top view, (c) Font view, (d) Ground plane layer.

as well as the thickness of the substrate and width of the shorting wall. Impedance bandwidth and gain of PIFA are certainly impacted by substrate's properties [21]. Generally, antennas use thin and high $\varepsilon_{r}$ substrates, which results in low gain and narrow bandwidth. Thus, these issues will directly affect the design of our proposed antenna.

\subsection{Geometric of reconfigurable antenna}

The base configuration 3-D model of the proposed antenna is depicted in Figure 2(a). The antenna consists 


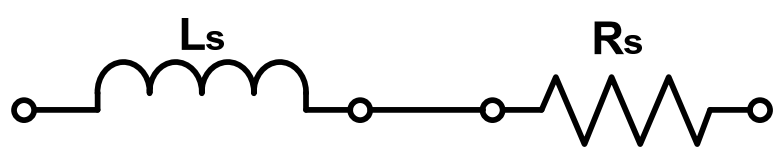

(a) Forward biased.

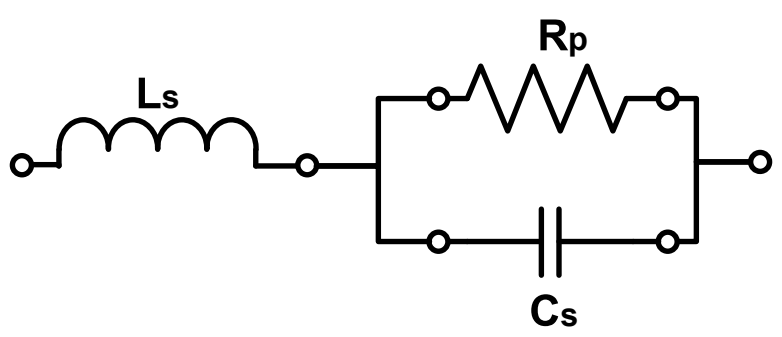

(b) Reverse biased.

Figure 3. PIN-diode's Equivalent circuit.

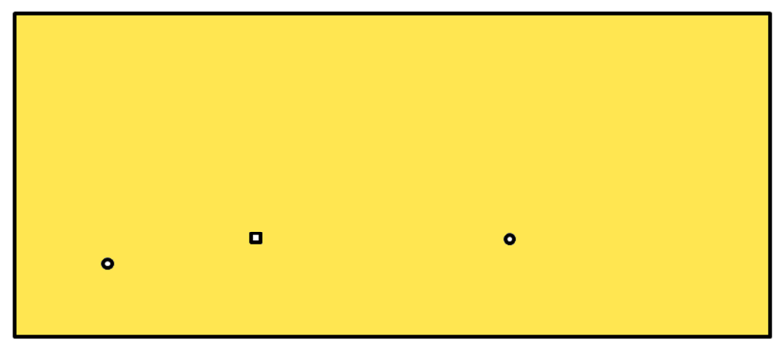

(a) Case 1- the ground plane is a uniform rectangular.

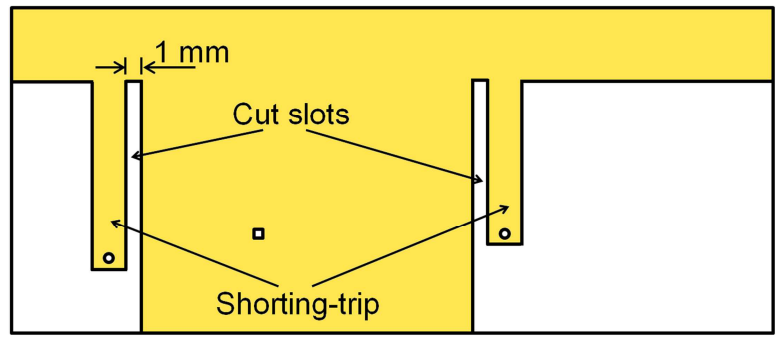

(b) Case 2- the ground plane uses the DGS.

Figure 4 . The cases to study the ground plane structure (top view).

of three layers: radiating patches, dielectric substrate, and ground plane. The radiating patches $(W p \times H p)$ and the ground plane $(\mathrm{Wg} \times \mathrm{Hg})$ are printed on both side of the dielectric substrate. The radiating patches include three parts: the main radiating element plate (P1) and two additional radiating elements (P2, P3), as exhibited in Figure 2(b). In Figure 2(c), two cylindrical shorting pins (radius $0.5 \mathrm{~mm}$ ) connect two additional radiators to the ground plane through a via-hole in the substrate. Similar, there is a feed gap cylinder of radius $0.5 \mathrm{~mm}$ between the main radiating element and the ground plane. The antenna is fed by a $50 \Omega$ SMA connector. The outer shield of the SMA connector is soldered to the surface of the ground plane. The DGS ground plane is represented in Figure 2(d). The radiating patches, shorting pins and ground plane use lossy copper material $(\sigma=5.8 \mathrm{MS} / \mathrm{m}$ and thickness $0.035 \mathrm{~mm})$.

In the design of the propose frequency reconfigurable antenna, we decide to use the FR4 dielectric substrate with thickness of $1.6 \mathrm{~mm}$, relative permittivity $\varepsilon_{r}$ of

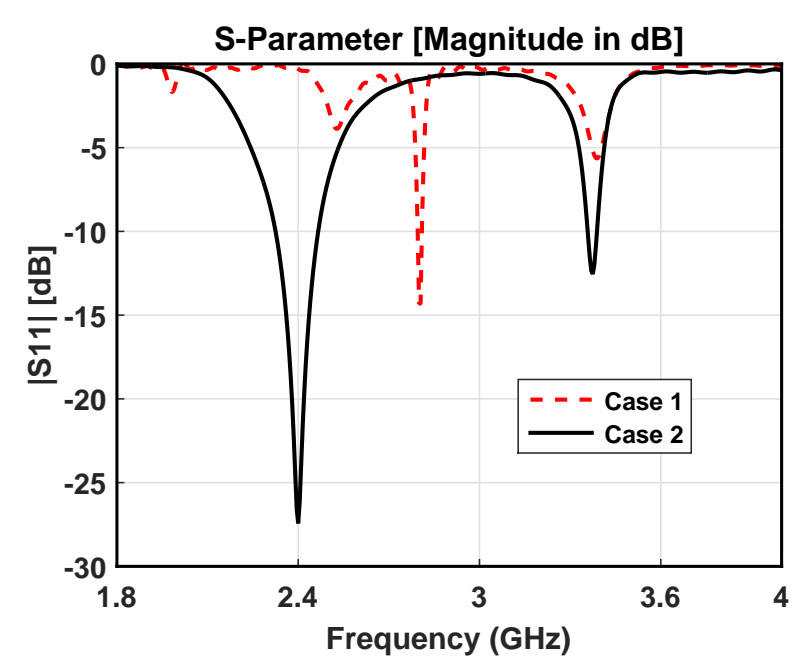

(a) The reflection coefficent.

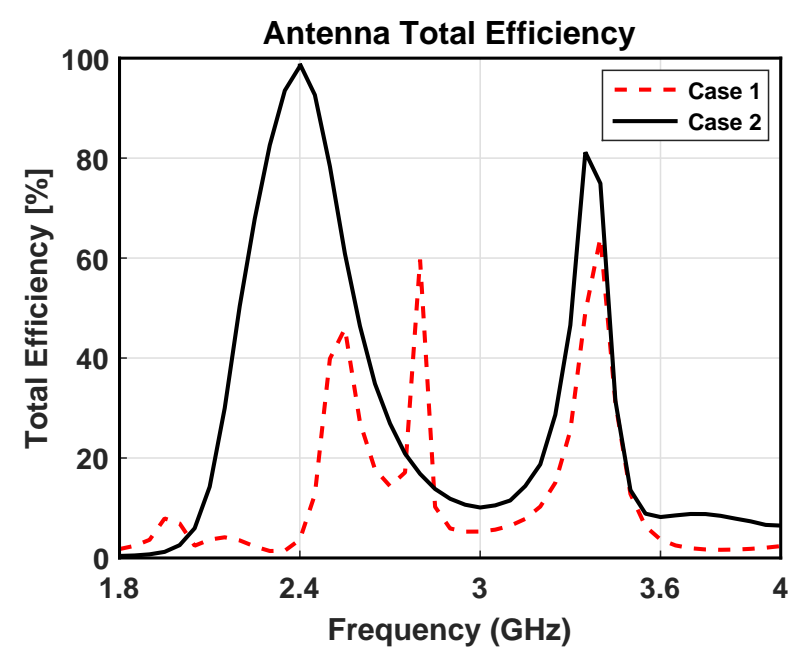

(b) The total efficiency.

Figure 5. The $1^{\text {st }}$ configuration - the antenna's results of various the ground plane structures show in Figure 4.

4.4 and a loss tangent of 0.02 , because this is a low cost common material. In basic reconfiguration, the operating frequency is assumed to be the center frequency of the WLAN band at $2.4 \mathrm{GHz}$. For the conditions of the operating frequency and the characteristics of dielectric substrate, the initial dimensions of the antenna are calculated from the formula (1) and (2). The proposed antenna's model is simulated using CST Microwave Studio.

As shown in Figure 2(b), three PIN-diodes (D1, D2 and D3) are located on the radiating patches. The PINdiodes are used to connect P1 to P2 and P3. When the proposed reconfigurable antenna is active, without changes in the shape or dimension of the radiating patches, the switching ON/OFF states of the PIN-diodes can modify the operating frequency of the antenna. Depending on the state of the three PIN-diodes, the proposed antenna can operate in eight different configurations. However, there are only three configurations which are useful. The $1^{\text {st }}$ configuration, when three PIN-diodes are on state OFF, the antenna has only the primary P1, as an IFA antenna which operates at 2.4 GHz. The $2^{\text {nd }}$ configuration, when D1 is on state 


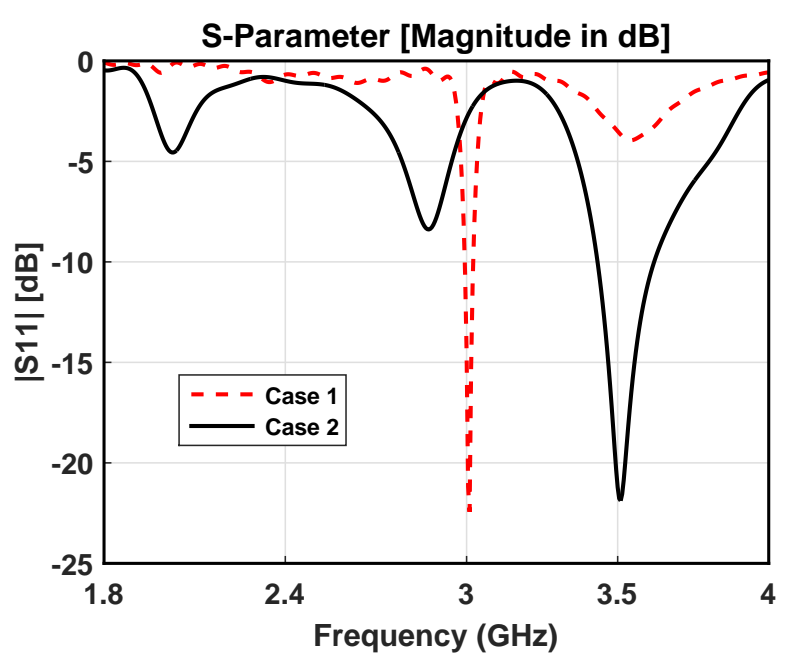

Figure 6 . The $2^{\text {nd }}$ configuration - the frequency responses of the Case 1 and Case 2 show in Figure 4

ON, D2 and D3 are both on state on OFF, this means the proposed antenna includes P1 and P2. Therefore, the proposed antenna becomes a PIFA antenna which operates at near $3.5 \mathrm{GHz}$. The $3^{\text {rd }}$ configuration, when all PIN-diodes are on state $\mathrm{ON}$, the proposed antenna consists all radiating patches. Thus, the proposed antenna radiates at $2.1 \mathrm{GHz}$, this is the lowest resonance frequency due to the largest size of antenna.

In this work, the PIN-diodes are Skyworks SMP1345079LF, the equivalent circuits model is illustrated in Figure 3. The circuit parameters are obtained from the datasheet where $L_{S}=0.7 \mathrm{nH}, R_{S}=1.5 \Omega, R_{P}=10 \mathrm{k} \Omega$, and $C_{S}=1.8 \mathrm{pF}$. Two capacitors $(\mathrm{C}=33 \mathrm{pF})$ are used as DC block. These capacitors are set in suitable positions to ensure that the DC supply for each PIN-diode does not affect the others.

\subsection{The defected ground structure}

The DGS is an etched periodic or non-periodic configuration defect in a ground plane. The DGS employs fewer slots or slits to modify the current distribution on the ground plane. As the result, the proposed reconfigurable antenna using DGS reduces size, adjustable resonant frequency and increases bandwidth.

In this work, the study of the ground plane structure is implemented in the $1^{\text {st }}$ configuration of the proposed antenna. Figure 4 shows the two cases to study the ground plane structure. Case 1 described in Figure 4(a), the initial ground plane is a uniform rectangular metal plate. Case 2 shown in Figure 4(b), the ground plane uses DGS, two cut open-end meander shapes are added on both sides of the ground plane. The two cut slots near the position of the sorting pins create the two shorting-trips. The shorting-trips connect the shorting pin to the ground. The lengths of shorting-trips depend on the position of the sorting pins. It means that we can increase the height of the shorting pin without changing the height of the proposed antenna.

In the $1^{\text {st }}$ configuration $(2.4 \mathrm{GHz})$, the antenna's operating frequencies for the different structure ground

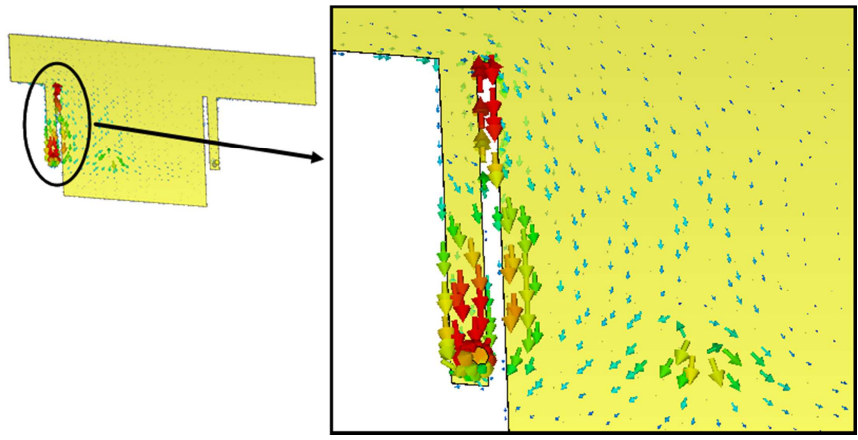

Figure 7. Simulate surface current distributions along to the cut slot of the ground plane layer (top view) at $3.5 \mathrm{GHz}$.

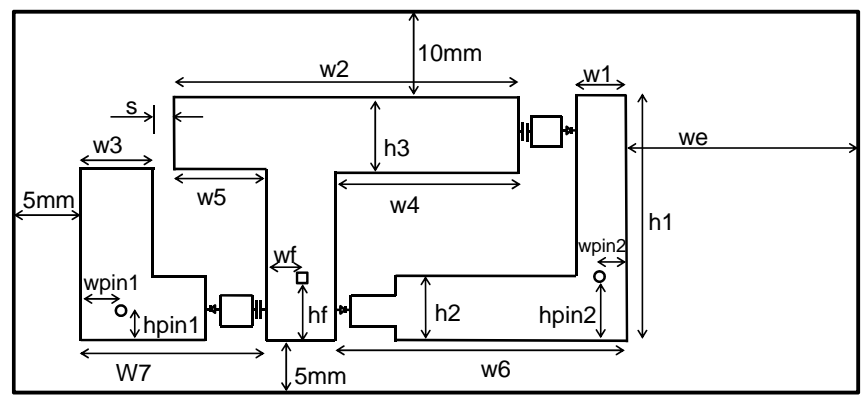

Figure 8. Dimension of the proposed PIFA antenna radiator patches.

plane cases are represented in Figure 5(a). The antenna using non-DGS (Case 1) operates at near 2.8 $\mathrm{GHz}$ with narrow bandwidth. Adding cut open-end meander shapes and slots (Case 2) have moved the resonant frequency downward to lower frequency, and also increase the bandwidth by approximately 10 times. Figure 5(b) shows the impact of the each case ground plane to total efficiency. The use of DGS has increased the performance of the antenna up to $98 \%$ compared with $60 \%$ while not using DGS.

When the proposed reconfigurable switches to $2^{\text {nd }}$ configuration, the antenna's operating frequency corresponding to the different ground plane structures are exhibited in Figure 6. In this configuration, PINdiode $\mathrm{D} 1$ is on state $\mathrm{ON}$, the $\mathrm{P} 1$ is connected to the P2, the proposed antenna is a PIFA antenna. As shown in Figure 6, the proposed antenna without DGS (Case 1) operates at near 3.0 GHz. In Case 2 (DGS), due to the impact of the left slot, the antenna operating frequency is adjusted to $3.5 \mathrm{GHz}$. Figure 7 shows a current passing through the left sorting pin and flow along to the left slot. Consequently, the antenna's operating frequency is strongly influenced by the length of slot between sorting-trip and the ground plane.

\subsection{Optimal parameters}

Figure 8 shows the dimension of the proposed reconfigurable antenna radiator patches's parameters. The $1^{\text {st }}$ configuration, $2^{\text {nd }}$ configuration and $3^{\text {rd }}$ configuration are assumed to operate at $2.4 \mathrm{GHz}, 3.5 \mathrm{GHz}$, and 2.1 GHz, respectively. These frequencies are directly influenced by the 18 parameters of the radiator patches, and all of them need to be optimized. Also note that 


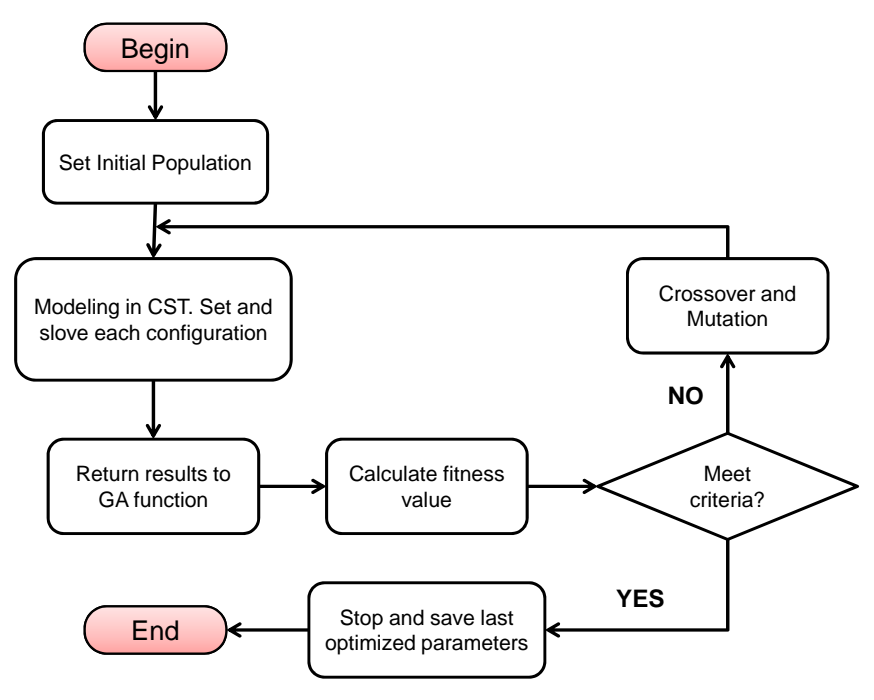

Figure 9. The flowchart of the GA.

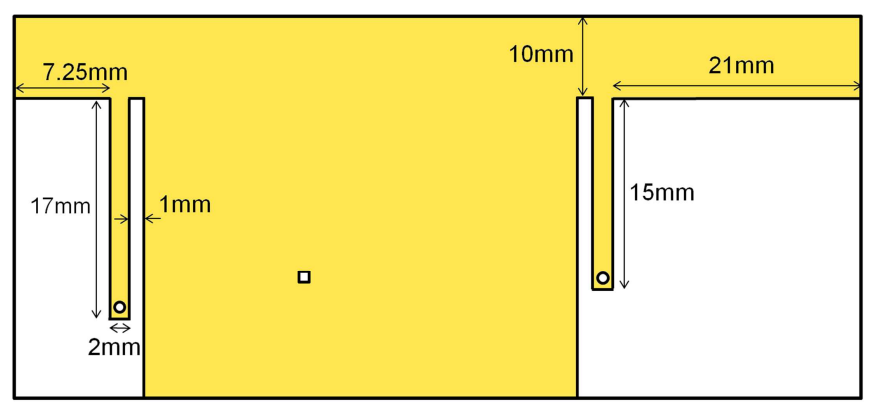

Figure 10. Dimension of the proposed PIFA antenna ground plane.

the proposed antenna has three configurations, most of the parameters can affect the performance of all configurations. Hence, the optimizing parameters cannot be performed separately on each configuration, and must be implemented concurrently with all configurations.

Compared to other optimization methods, the performance of the GA is not higher when all algorithms are allowed equal computation time [22]. However, the GA algorithm has a faster execution time while all algorithms using the same parameters setting and optimization problems [23]. On the other hand, the GA algorithm is well suited for antenna design [24]. Therefore, in this work, the GA is used to optimize the parameters. The GA is a stochastic search method which operates based on the mechanics of population genetics and selection [24]. As described in Figure 9, the optimization GA model in this design is program by Visual Basic for Application language which embedded in to CST and summarized as follows:

Step 1: All 18 parameters are genes. Each parameter is encoded in the binary string. The chromosome is constructed from all the binary strings of parameters. After that, the initial population is created randomly. Determine a logical reason for the start of optimization. From there, identify parameter values for the chromosome.

Step 2: Depending on values of the decoded parameters, create 3-D structural of the proposed antenna
Table I

Antenna Dimensions (UNIT: MM)

\begin{tabular}{|l|l|l|l|l|l|}
\hline \hline$w 1$ & 4 & $w 7$ & 12.5 & $h f$ & 4 \\
\hline$w 2$ & 39.5 & $w e$ & 20 & $w f$ & 3 \\
\hline$w 3$ & 6.5 & $h 1$ & 18.5 & hpin1 & 2 \\
\hline$w 4$ & 15 & $h 2$ & 4 & wpin1 & 3.75 \\
\hline$w 5$ & 4 & $h 3$ & 6 & hpin 2 & 4 \\
\hline$w 6$ & 21 & $s$ & 2 & wpin2 & 2 \\
\hline \hline
\end{tabular}

and solve the model in CST software with each the antenna's configuration. CST returns values of $\left|S_{11}\right|$ correspond to the assumed frequency of each configuration $(2.4 \mathrm{GHz}, 3.5 \mathrm{GHz}$, and $2.1 \mathrm{GHz})$. The design goal is the configurations of the proposed antenna will operate respectively at 2.4, 3.5 and $2.1 \mathrm{GHz}$.

Step 3: GA can begin to perform optimally. The fitness function is used to assign a fitness value to each of the individuals in the GA population. The fitness value for each individual is calculated using the input reflection coefficient $\left|S_{11}\right|$ parameters from CST and the fitness function (3):

$$
\text { fitness }=\frac{\sum_{N}^{1}\left|S_{11}\left(f_{i}\right)\right|}{N}
$$

where $N$ is the number of evaluated configurations, $f_{i}$ is the predetermined frequency of $\mathrm{i}^{\text {th }}$ configuration, $\left|S_{11}\right|$ is the magnitude of the reflection coefficient at the $i^{\text {th }}$ frequency.

Step 4: Based on the individuals is optimized by step 3, the next generation is selected, crossover and mutation operators. This process is performed iterative until the fitness value is converges (Optimal) or a termination criteria is met.

After the implementation of the optimization of GA, the antenna's parameters are determined as shown in Table I. The ground plane is DGS with the position of the cut shapes based on the position of the two shorting pins. Therefore, the values of the radiator patches' parameters will determine the dimensions of the ground plane as exhibited in Figure 10.

\subsection{Surface current distribution}

In the $1^{\text {st }}$ configuration, the proposed antenna operates with only the P1 radiator when all of the PIN-diodes are off. As shown in Figure 11(a), the strong current distribute from the feed position to the right side of Tshape $\mathrm{P} 1$ radiator plate. This implies that the resonant length $(h 1-h f+w f+w 4)$ is close to the quarter-wavelength of $2.4 \mathrm{GHz}$.

In the $2^{\text {nd }}$ configuration, D1 PIN-diode is on, the P1 radiator plate is connected to the $\mathrm{P} 2$ radiator plate. As seen from Figure 11(b), the strong current distribution appears from the feed position to the location of the right shorting pin and ends at the top edge of the P2 radiator plate. There is also a current distribution from the feed position to the upper right edge of the P1 radiator plate. This demonstrates the form of current distribution is like a dipole. It is clearly observed that resonant length $(h 1+w f+w 7-w p i n 1+h 1-h 3)$ is $42.8 \mathrm{~mm}$. 


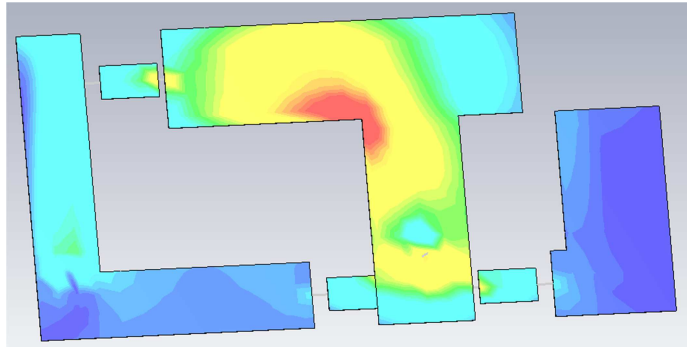

(a) $2.4 \mathrm{GHz}$.

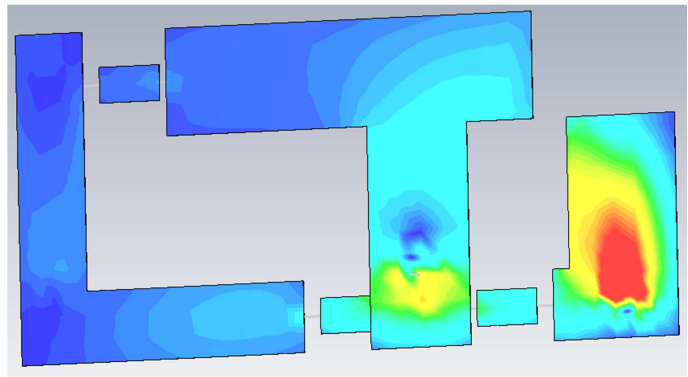

(b) $3.5 \mathrm{GHz}$.

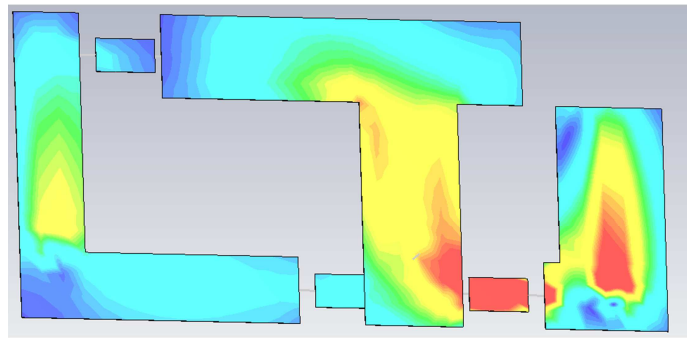

(c) $2.1 \mathrm{GHz}$

Figure 11. Surface current distribution (bottom view).

This exhibits a resonant length of half-wavelength at 3.5 GHz.

Fig. 11(c) shows the current distribution of the proposed antenna in the $3^{\text {rd }}$ configuration where all PINdiodes are on. The radiator patches are connected through three PIN-diodes. These connections make the current distributions exist at all of three radiator plates. Hence, the electrical length of the $3^{\text {rd }}$ configuration is longer than the $2^{\text {nd }}$ configuration. As a result, the operating frequency of the $2^{\text {nd }}$ configuration shifts to lower frequency and becomes $2.1 \mathrm{GHz}$. On the other hand, the maximum currents distribute at the feeding point and two shorting pins, and the null point is top stub position between the P1 and P2 radiator plates. That mean the $3^{\text {rd }}$ configuration has resonant length of a wave-length at $2.1 \mathrm{GHz}$.

\section{Simulated and Measured Results}

The three PIN-diodes (D1, D2 and D3) afford three possible and useful switching configurations. The CST studio software is used to conduct the simulation on the impedance bandwidth $(-10 \mathrm{~dB})$ of the propose antenna in three different configurations, as demonstrated in Fig. 12. In the $1^{\text {st }}$ configuration, all PIN-diodes are OFF, only $\mathrm{P} 1$ radiator plate is functioning. The propose an-

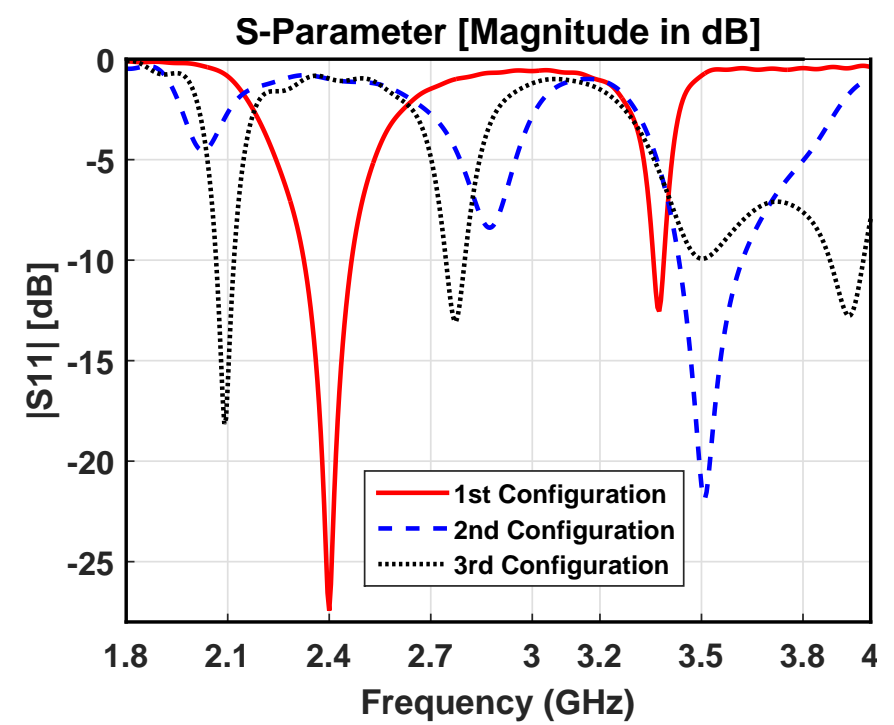

Figure 12. Simulated S11 response the three configurations of the proposed antenna.

Table II

The Operating Results of the Proposed Reconfigurable PIFA ANTENNA

\begin{tabular}{l|cccc}
\hline \hline Configuration & $\begin{array}{c}\text { Frequency } \\
\text { (GHz) }\end{array}$ & $\begin{array}{c}\text { Gain } \\
\text { (dBi) }\end{array}$ & $\begin{array}{c}\text { Total } \\
\text { efficiency } \\
(\mathbf{\%})\end{array}$ & $\begin{array}{c}\text { Bandwidth } \\
\text { (\%) }\end{array}$ \\
\hline 1st Configuration & 2.4 & 3.55 & 98.3 & 6.3 \\
\hline 2nd configuration & 3.5 & 4.33 & 96.3 & 5.4 \\
\hline 3rd configuration & 2.1 & 0.48 & 57 & 2.4 \\
\hline \hline
\end{tabular}

tenna operates at $2.4 \mathrm{GHz}$ with impedance bandwidth $(-10 \mathrm{~dB})$ of $6.3 \%$. Hence, the $1^{\text {st }}$ configuration is suitable for WLAN 2.4 GHz applications. When D1 PIN-diode is $\mathrm{ON}$ and the remaining PIN-diodes are OFF, the proposed antenna switches to the $2^{\text {nd }}$ configuration. In this configuration, the antenna becomes a PIFA antenna which operates at $3.5 \mathrm{GHz}$ with impedance bandwidth of $5.4 \%$. Accordingly, the m-WiMAX band is covered. In the $3^{\text {rd }}$ configuration, all of radiator plates are radiating, the antenna operating frequency is shifted to $2.1 \mathrm{GHz}$ with impedance bandwidth of $2.4 \%$. This configuration can serve UTMC $2.1 \mathrm{GHz}$ applications. All configurations of the proposed antenna have VSWR $<2$ at desired frequency bands.

Table II summarizes the simulated results of the proposed frequency reconfigurable antenna. The antenna gain is $3.55 \mathrm{dBi}$, and the total efficiency is $98.3 \%$ in the $1^{\text {st }}$ configuration $(2.4 \mathrm{GHz})$. When the proposed antenna changes to the $2^{\text {nd }}$ configuration, as the frequency increased, the highest gain is $4.33 \mathrm{dBi}$ at $3.5 \mathrm{GHz}$ with total efficiency of $96.3 \%$. However, in $3^{\text {rd }}$ configuration, the obtained gain and total efficiency are the lowest at $0.48 \mathrm{dBi}$ and $57 \%$, respectively. The reason is as follows. When all PIN-diodes are on state ON, the loss of PINdiodes increases the loss of the antenna. On the other hand, the antenna structure presents a combination of folded loop-antenna and a PIFA which made the an- 

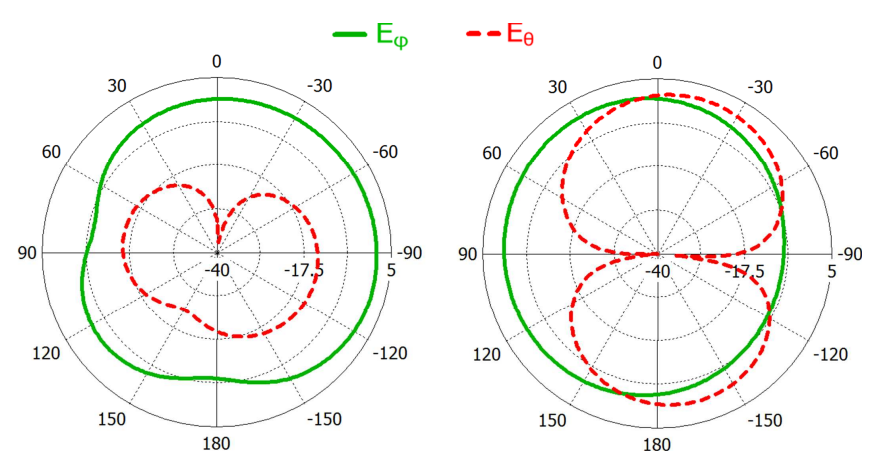

(a) The $1^{\text {st }}$ configuration - $2.4 \mathrm{GHz}$.
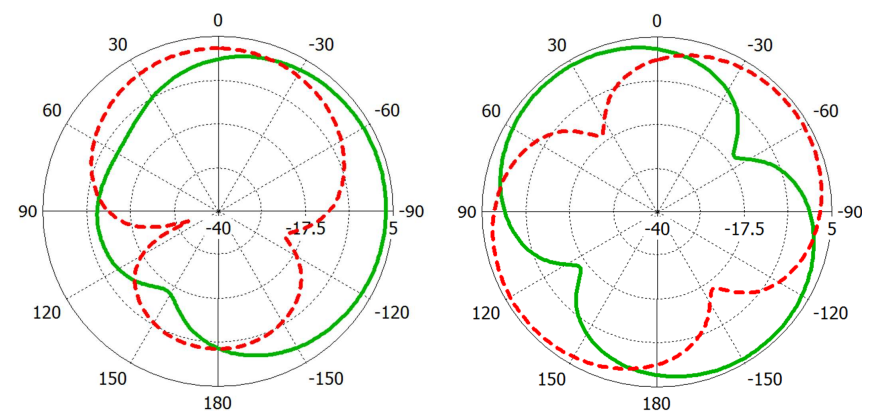

(b) The $2^{\text {nd }}$ configuration $-3.5 \mathrm{GHz}$.
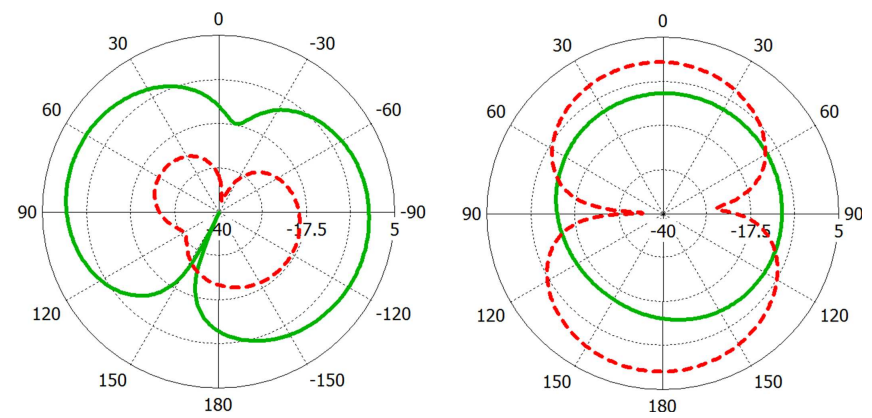

(c) The $3^{\text {rd }}$ configuration $-2.1 \mathrm{GHz}$.

Figure 13. Simulated radiation patterns in the $x y-$ and $x z-$ planes.

tenna has smaller radiation resistances than loss resistances. These reasons significantly reduce efficiency and gain of the antenna. Fig. 13 shows simulated radiation patterns of the proposed reconfiguration antenna at 2.4, 3.5 , and $2.1 \mathrm{GHz}$ for the $1^{\text {st }}, 2^{\text {nd }}$, and $3^{\text {rd }}$ configuration, respectively. The patterns are presented in the $x y-$ and xz- planes according to Fig. 2(a). We can see clearly that the radiation pattern in all planes are rather smooth half-wavelength dipole-like characteristics.

To validate the design results, a prototype of the proposed antenna with optimize geometrical parameters (shows in TABLE I) is manufactured and measured. As show in Figure 14, the proposed reconfigurable PIFA antenna is fabricated on FR4 dielectric substrate with a permittivity of 4.4 and a dimension of $64.5 \times$ $33.5 \times 1.6 \mathrm{~mm}^{3}$. The radiator patches have dimension of $39.5 \times 18.5 \times 0.035 \mathrm{~mm}^{3}$. The input reflection coefficients $\left|S_{11}\right|$ in all configurations have been measured using Keysight PNA-X vector network analyzer. The state ON of the PIN-diodes is controlled by the DC bias, which is connected to the PIN-diodes with a forward voltage of $5 \mathrm{~V}$ and a forward bias current of $10 \mathrm{~mA}$. The measured and simulated reflection coefficient results are

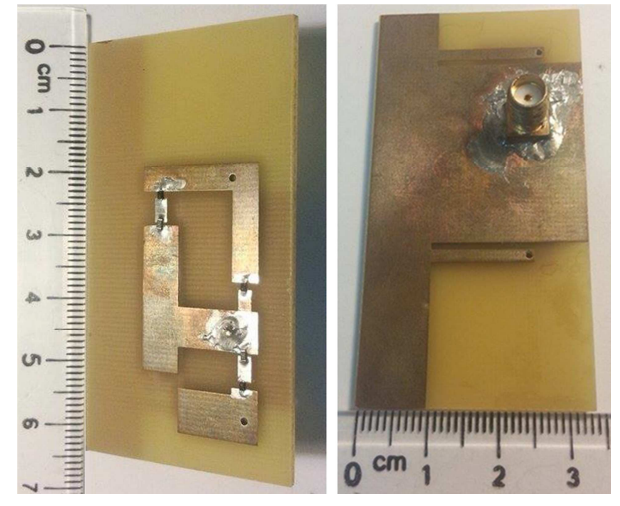

Figure 14. Prototype of the proposed antenna.

illustrated in Figure 15 for comparison. In the $1^{\text {st }}$ configuration, the measured impedance bandwidth $(-10 \mathrm{~dB})$ is approximately $7.4 \%(2.2-2.39 \mathrm{GHz})$. The measured impedance bandwidth is approximately 5.5\% (3.37-3.56 $\mathrm{GHz}$ ) in $2^{\text {nd }}$ configuration. When the proposed antenna operates in $3^{\text {rd }}$, the measured impedance bandwidth is $2.7 \%(2.031-2.104 \mathrm{GHz})$. There are slightly discrepancies between the simulated and measured results, which could be attributed to the accuracy of fabrication, the effect of material and the quality of PIN-diodes.

It is noted in our proposed antenna structure that by using DGS and optimal antenna parameters, the overall dimension of the proposed antenna occupies a $66 \%$ smaller volume in comparison with our previously work [18]. More specifically, our antenna structure has a total size of $64.5 \times 33.5 \times 1.6 \mathrm{~mm}^{3}$, while that in [18] is $42.5 \times 80 \times 3 \mathrm{~mm}^{3}$. The use of DGS also increases the impedance bandwidth of the antenna. The antenna proposed in [18] operates at $2.4 \mathrm{GHz}$ and $3.5 \mathrm{GHz}$ with the bandwidth of $2.6 \%$ and $2.1 \%$, respectively. In this study, the impedance bandwidth at $2.4 \mathrm{GHz}$ and $3.5 \mathrm{GHz}$ reaches $6.3 \%$ and $5.4 \%$, respectively. Besides, it is interesting to see that, the antenna proposed in [18] has a complex structure (including four layers: the radiating patches, the dielectric substrate, the air, and the ground plane), while ours is simpler thanks to removing the air layer. Moreover, the radiating patches and the ground plane are printed on both surfaces of a dielectric substrate, thus increasing the precision in the fabrication process.

\section{Conclusions}

In this paper, a low profile frequency reconfigurable PIFA antenna design with three PIN-diodes and using defected ground structure has been presented to cope with the frequency bands of WLAN, m-WiMAX, and UMTS. The DGS technique with sorting-trip is used to adjust downward frequency without increasing the thickness of the antenna. The GA algorithm is used simultaneously on all possible configurations for the antenna parameters selection. The frequency bands of the proposed antenna are controlled by switching the states of three PIN-diodes. Depending on operating 


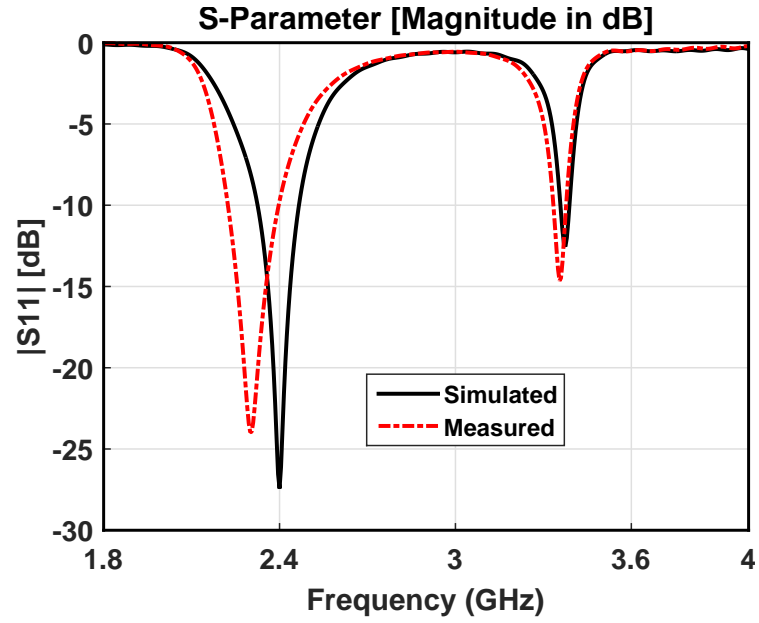

(a) $1^{\text {st }}$ configuration.

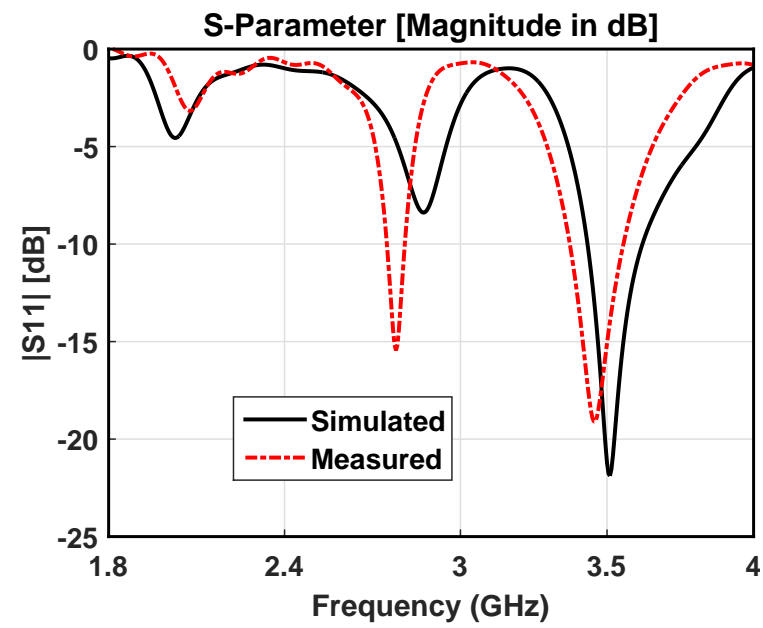

(b) $2^{\text {nd }}$ configuration.

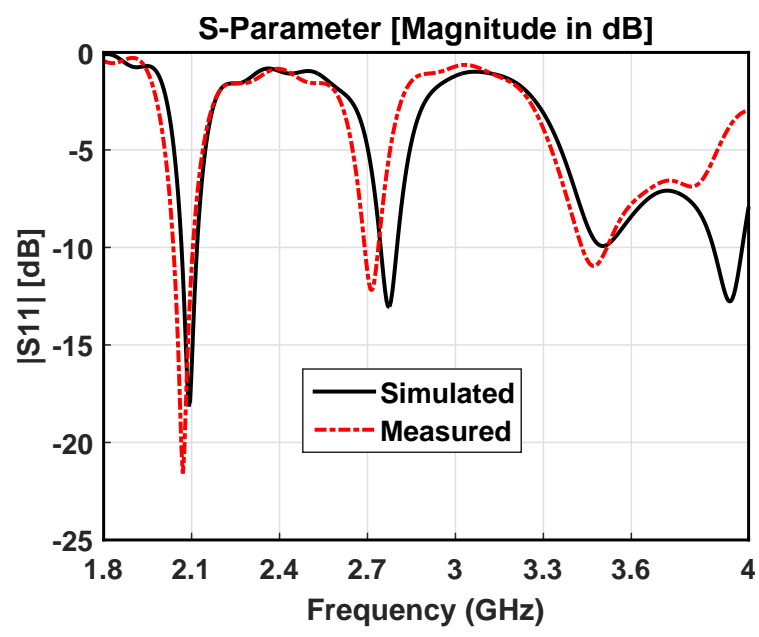

(c) $3^{\text {rd }}$ configuration.

Figure 15. Simulated and measured results of the proposed reconfiguration PIFA antenna.

configurations, the proposed antenna operates at 2.4 $\mathrm{GHz}, 3.5 \mathrm{GHz}$, and $2.1 \mathrm{GHz}$ with gains of $3.55 \mathrm{dBi}$, $4.33 \mathrm{dBi}$, and $0.48 \mathrm{dBi}$, respectively. The efficiencies for the $1^{\text {st }}, 2^{\text {nd }}$, and $3^{\text {rd }}$ configurations are $98.3 \%, 96.3 \%$, and $57 \%$, respectively. The measurement results are matched with the simulation ones, while the design can be seen to be compact, low profile, lightweight, simple structure and easy to fabricate.

\section{REFERENCES}

[1] M. S. Ahmad, C. Y. Kim, and J. G. Park, "Multishorting Pins PIFA Design for Multiband Communications," International Journal of Antennas and Propagation, vol. 2014, pp. 1-10, Feb. 2014.

[2] C. G. Christodoulou, Y. Tawk, S. A. Lane, and S. R. Erwin, "Reconfigurable Antennas for Wireless and Space Applications," Proceedings of the IEEE, vol. 100, no. 7, pp. 2250-2261, Jul. 2012.

[3] J. Costantine, Y. Tawk, S. E. Barbin, and C. G. Christodoulou, "Reconfigurable Antennas: Design and Applications," Proceedings of the IEEE, vol. 103, no. 3, pp. 424-437, Mar. 2015.

[4] K. R. Boyle and P. G. Steeneken, "A Five-Band Reconfigurable PIFA for Mobile Phones," IEEE Transactions on Antennas and Propagation, vol. 55, no. 11, pp. 3300-3309, Nov. 2007.

[5] S. Jegadeesan, Z. Mansouri, A. Veeramani, and F. B. Zarrabi, "Ultra Wideband PIFA Antenna with Supporting GSM and WiMAX for Mobile Phone Applications," in Fifth International Conference on Advanced Computing $\mathcal{E}$ Communication Technologies (ACCT), Feb. 2015, pp. 15-20.

[6] C. H. See, H. I. Hraga, R. A. Abd-Alhameed, N. J. McEwan, J. M. Noras, and P. S. Excell, "A Low-profile Ultra-wideband Modified Planar Inverted-F Antenna," IEEE Transactions on Antennas and Propagation, vol. 61, no. 1, pp. 100-108, 2013.

[7] Jong-Hyuk Lim, Gyu-Tae Back, Young-Il Ko, ChangWook Song, and Tae-Yeoul Yun, "A Reconfigurable PIFA Using a Switchable PIN-Diode and a Fine-Tuning Varactor for USPCS/WCDMA/m-WiMAX/WLAN," IEEE Transactions on Antennas and Propagation, vol. 58, no. 7, pp. 2404-2411, Jul. 2010.

[8] M. Unlu, H. Mopidevi, A. Zohur, and B. A. Cetiner, "Frequency Reconfigurable, 220-5000 MHz, Five-band RF MEMS PIFA," in IEEE 55th International Midwest Symposium on Circuits and Systems (MWSCAS), Aug. 2012, pp. 65-68.

[9] T. D. Nguyen, Y. Duroc, V. Y. Vu, and T. P. Vuong, "Novel Reconfigurable 8 - Shape PIFA Antenna using PIN Diode," in International Conference on Advanced Technologies for Communications (ATC), Aug. 2011, pp. 272-275.

[10] Z.-J. Jin, J.-H. Lim, and T.-Y. Yun, "Frequency Reconfigurable Multiple-input Multiple-output Antenna with High Isolation," IET Microwaves, Antennas \& Propagation, vol. 6, no. 10, p. 1095, 2012.

[11] Y. Sung, "Multi-band Reconfigurable Antenna for Mobile Handset Applications," IET Microwaves, Antennas $\mathcal{E}$ Propagation, vol. 8, no. 11, pp. 864-871, Aug. 2014.

[12] C.-H. Chang and K.-L. Wong, "Printed $\lambda / 8$-PIFA for Penta-Band WWAN Operation in the Mobile Phone," IEEE Transactions on Antennas and Propagation, vol. 57, no. 5, pp. 1373-1381, May 2009.

[13] H. Rajagopalan, J. M. Kovitz, and Y. Rahmat-Samii, "MEMS Reconfigurable Optimized E-Shaped Patch Antenna Design for Cognitive Radio," IEEE Transactions on Antennas and Propagation, vol. 62, no. 3, pp. 1056-1064, Mar. 2014

[14] A. Patnaik, D. Anagnostou, C. G. Christodoulou, and J. C. Lyke, "Modeling Frequency Reconfigurable Antenna Array using Neural Networks," Microwave and optical technology letters, vol. 44, no. 4, pp. 351-354, 2005.

[15] A. Akdagli, K. Guney, and B. Babayigit, "Clonal Selection Algorithm for Design of Reconfigurable Antenna Array with Discrete Phase Shifters," Journal of Electromagnetic Waves and Applications, vol. 21, no. 2, pp. 215-227, Jan. 2007.

[16] S. Song and R. D. Murch, "An Efficient Approach for Optimizing Frequency Reconfigurable Pixel Antennas 
Using Genetic Algorithms," IEEE Transactions on Antennas and Propagation, vol. 62, no. 2, pp. 609-620, Feb. 2014.

[17] T. D. Nguyen, Y. Duroc, V. Y. Vu, and T. P. Vuong, "Genetic Algorithm for Optimization of L-Shaped PIFA Antennas," International Journal of Microwave and Wireless Technologies, vol. 3, no. 06, pp. 691-699, Dec. 2011.

[18] P. T. Minh, T. T. Thao, N. T. Duc, and V. V. Yem, "A Novel Multiband Frequency Reconfigurable PIFA Antenna," in International Conference on Advanced Technologies for Communications (ATC), Oct. 2016, pp. 7-12.

[19] J. Liu, W. Yin, and S. He, "A New Defected Ground Structure and Its Application for Miniaturized Switchable Antenna," Progress In Electromagnetics Research, vol. 107, no. May, pp. 115-128, 2010.

[20] Y.-T. Jean-Charles, V. Ungvichian, and J. A. Barbosa, "Effects of Substrate Permittivity on Planar InvertedF Antenna Performances," Journal of Computers, vol. 4, no. 7, pp. 610-614, 2009.

[21] A. P. Dabhi and S. K. Patel, "Response Of Planar Inverted F Antenna Over Different Dielectric Substrates," International journal of scientific $\mathcal{E}$ technology research, vol. 3, no. 5, pp. 114-117, 2014

[22] M. A. Panduro, C. A. Brizuela, L. I. Balderas, and D. A. Acosta, "A Comparison of Genetic Algorithms, Particle Swarm Optimization and the Differential Evolution Method for the Design of Scannable Circular Antenna Arrays," Progress In Electromagnetics Research B, vol. 13, pp. 171-186, 2009.

[23] S. P. Lim and H. Haron, "Performance comparison of Genetic Algorithm, Differential Evolution and Particle Swarm Optimization towards benchmark functions," in IEEE Conference on Open Systems (ICOS), Dec. 2013, pp. 41-46.

[24] R. L. Haupt, "An Introduction to Genetic Algorithms for Electromagnetics," IEEE Antennas and Propagation Magazine, vol. 37, no. 2, pp. 7-15, 1995.

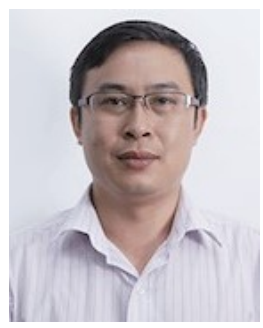

Pham Trung Minh was born in Hai Phong, Vietnam, in 1980. He received the Master of Engineering in 2007 from the Faculty of Information Technology, Military Technical Academy, Vietnam. Currently, he is a Ph.D candidate at Dept. of Telecommunication Systems, School of Electronics and Telecommunications, Hanoi University of Science and Technology, Vietnam. His research interests are microstrip antennas and antenna array and embedded systems signal processing, intelligence control system

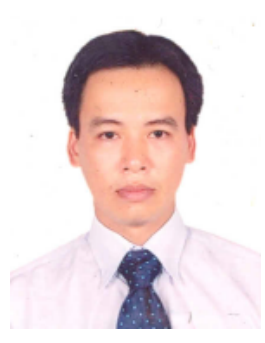

Nguyen Trong Duc received the Ph.D. degree in communications from Laboratory LAHC, Institute Microelectronic Electromagnetic and Photonic, University of Grenoble, France in 2012. Currently, he is the lecturer of the Faculty of Information Technology, Vietnam Maritime University. Dr. Nguyen Trong Duc's areas of expertise are multi-antenna communication and localization systems, microstrip antennas and antenna array signal processing, embedded systems and intelligence control

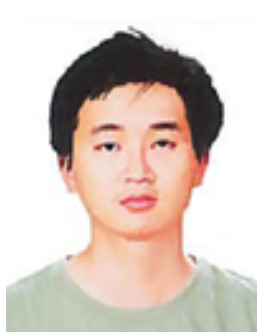

Phan Xuan Vu received the B.S. degree in computer science and the M.S. degree in Intelligent and Communication Systems from the University of Cergy-Pontoise, France, in 2008 and 2010, respectively, and the PhD degree in Signal Processing from the University of Rennes 1 and University of Grenoble, France, in 2014. He is currently working as a lecturer-researcher in the School of Electronics and Telecommunications, Hanoi University of Science and Technology, Vietnam. His research interests include microwave engineering, antenna and propagation, radar signal processing, and large scale RFID.

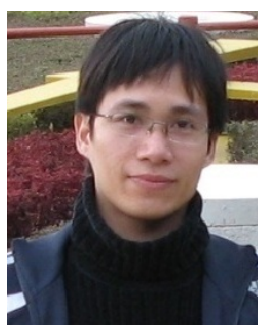

Nguyen Thanh Chuyen received the M.S. degree in communications engineering from $\mathrm{Na}-$ tional Tsing-Hua University, Taiwan, in 2008, and the Ph.D. degree in informatics from Kyoto Univer- sity, Japan, in 2013. In 2014, he was a Visiting Researcher with The University of Aizu, Japan. He is currently an Assistant Professor with the School of Electronics and Telecommunications, Hanoi University of Science and Technology, Vietnam. His research interests include statistics, optimization algorithms and their applications in wireless communication systems. He received the Fellow award from the Hitachi Global Foundation in 2016.

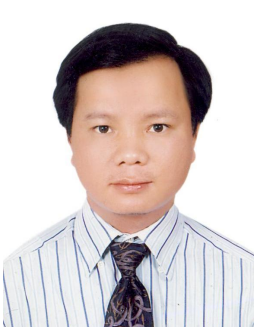

Vu Van Yem was born in 1975 in Hai Phong, Vietnam. He received the Ph.D. degree in communications from Department of Electronics and Communications, TELECOM ParisTech (formerly ENST Paris) France in 2005. From 2006 to 2007, he was a postdoctoral researcher at the Department of Hyper-frequencies and Semiconductor, Institute of Electronics, Microelectronics and Nanotechnology (IEMN) France. He has been qualified to be named as Associate Professor since November, 2009.

Currently, he is the Deputy - Dean of Graduate School and the Head of the Department of Telecommunication Systems, School of Electronics and Telecommunications, Hanoi University of Science and Technology, Vietnam. His area of expertise are microwave engineering, antenna, chaos-based digital communications as well as wireless communication and localization systems. 